\title{
A NEW BACILLUS PATHOGENIC TO FISH.
}

\section{By R. Greig Smith, M.Sc., Macleay Bacteriologist.}

\section{(Plates viii.-ix.)}

A few fishes which were supposed to have died of some obscure disease were received from the Fisheries Department towards the end of March. They came from Lake Illawarra, an inlet from the sea about 33 miles south from Sydney. The flesh of the fishes was of a greenish hue and somewhat congested in the neighbourhood of the main blood vessels. The vessels of the stomach and intestine were also much congested, but beyond these appearances there was nothing abnormal to be seen. When, however, portions of the various muscles and organs were examined microscopically a large rod-shaped bacterium with rounded ends was invariably found in all the fishes. In some cases the organisms had completely filled the smaller blood vessels. Portions of fluid, juice or pulp from the parts examined gave rise, when inoculated into culture media, to many bacterial colonies, among which those containing the large rod-shaped organism predominated. The organism readily produced spores, and accordingly by the nomenclature of Lehmann and Neumann and others it is a bacillus.

In order to test the pathogenicity of the bacillus, an agar culture which had partly sporulated was distributed in 4 c.c. of normal saline and 0.5 c.c. injected under the skin of a guinea-pig. No reaction followed, and the animal remained in health.

It now remained to test its pathogenicity to fish, and with this object in view three fishes comprising a White Trevally, Caranx georgianus, C. \& V., a large and a small Mullet, Mugil sp., were inoculated with 0.1 to 0.3 c.c. of a similar suspension of bacilli and spores subcutaneously in the left upper dorsal region between the median fin and the tail. In 24 hours the movements of the fishes had become sluggish. The trevally showed a white patch, from which the scales had fallen, stretching for about an inch and a half forwards and backwards from the point of inoculation. 
It died towards the end of the second day. The two mullets developed reddish discoloured patches at first on an area around the point of inoculation and afterwards further forward on that side and also on the other. The scales fell off these places and the body became swollen. The smaller mullet died in 45 , the larger in about 100 hours.

In the post mortem examination of the trevally the muscle beneath the epidermal scar was seen to be of a cream-white colour and brittle, both in appearance and texture like boiled cod roe. Portions of this when pressed between cover-glasses were found to be swarming with motile bacilli. The muscle on the side reverse to that inoculated was apparently healthy and free from bacteria. The blood vessels were congested. The peritoneal cavity contained a quantity of yellow serous fluid in which floated large white cheese-like masses. The liver was pale and mottled, the gall bladder distended, the stomach slightly congested. The kidneys, spleen and heart were apparently normal. The intestine, especially in the posterior portion, was much congested. A number of inoculations were made on sloped agar and these showed the organisms in pure culture in the heart blood, the gangrenous muscle near the site of inoculation and the apparently healthy muscle on the reverse side. The liver and peritoneal fluids gave negative results.

The smaller mullet showed very few lesions. The intestine was congested and filled with gas, the liver was dark and mottled. The other organs were apparently normal, and the muscle, although discoloured near the point of inoculation, did not show the localised gangrenous appearances of the other fishes. Pure cultures of the organism were obtained from the liver, the heartblood and the muscle from the inoculated as well as the reverse side.

The larger mullet showed several hæmorrhagic areas over the surface of the epidermis. The scales had fallen from places, revealing patches of a purplish to grey-blue tinge with reddish streaks. The muscles had three gangrenous spots, one at the site of inoculation, another further forward on the reverse side 
and near the epidermis, while a third was found deep down in the tissue near the vertebral column on the reverse side and about midway between the first two lesions. The peritoneal cavity was distended with a quantity of a whitish fluid. The intestines were swollen with gas and had the blood-vessels congested. The liver was mottled, the gall-bladder distended. The blood vessels of the stomach were slightly congested; those inside the swim bladder showed much congestion. The heart, kidneys and spleen appeared normal. The colour of the muscles generally was bluish with a pink tinge. The gangrenous portions of the muscles were cream-white and brittle as in the case of the trevally, and they swarmed with bacilli. When small portions of the heart-blood and of the three gangrenous localities were inoculated into liquefied culture media, there were obtained colonies of the bacillus and no other organism. The spleen and peritoneal fluids contained a mixture of organisms, including the bacillus. The liver was sterile.

\section{Characteristics of the Organism.}

Microscopical appearance.-The bacilli appear as large rods, with rounded ends, and measure $0 \cdot 8: 2-3 \cdot 6 \mu$ in bouillon culture. They grow singly, in pairs, and short chains in liquid media. On solid media they grow as long chains or filaments, which lie parallel with one another. The cells show, when stained with methylene-blue, a granular structure. They stain deeply by Gram's method.

Spores. - The rod easily forms a central spore, at first round, then becoming oval, and finally measures $1: 1 \cdot 5-1 \cdot 6 \mu$. Although the spore is broader than the bacillus, the latter does not assume a marked clostridium appearance, owing to the remains of the rod broadening as sporulation proceeds.

Motility.-The younger bacilli in bouillon are actively motile, the motion being fish-like. The older bacilli and those grown on solid media either move extremely slowly or are non-motile. The flagella are terminal or irregularly distributed over the surface; in most cases there is a single flagellum at one end of the rod. 
Relation to media, temperature and oxygen.-The organism grows quickly in the usual culture media at $20^{\circ}$ as well as at $37^{\circ}$. In the absence of air, the growth is very scanty indeed.

Gelatine plate.-The colonies appear at first as white circular points, which develop into circular colonies. The medium slowly liquefies, forming a shallow, saucer-like depression, in which the colony assumes a more or less zonal or annular appearance. There may be a white centre, around which are arranged circles or zones of varying intensity of white, or the centre may be clear, the margin clear, and midway between centre and margin a broad ring, which may appear homogeneous or striped with radial bands. As liquefaction proceeds the growth gathers towards the centre and the margin, when an appearance is presented like that figured in Lehmann and Neumann's Diagnostik, i. 3i, iv. This stage is reached in about 4 days, at $22^{\circ} \mathrm{C}$.

When examined with a 60 -fold magnification, the colonies near the surface and deep in the medium are circular, granular and brownish-black in colour, with a rough margin as if beset with short hairs. In thickly sown plates the deep colonies are irregular and generally studded with opaque root-like fibres. The colonies absolutely on the surface spread at first irregularly like a fragment of twisted or crumpled paper. The rosette-like colony then sends out straight and sinuous granular processes, which are visible as long as liquefaction has not proceeded very far. At the later stages, when the colony has assumed the annular appearance, the structure is seen to consist of tufts of short interlacing threads.

Gelatine stab. At first there is an uncharacteristic white filiform growth along the track made by the inoculating needle. The growth widens a little more at the surface than in the depth; an air-bubble then appears at the top of the canal. Liquefaction proceeds in the upper layers of the gelatine, producing a funicular or napiform liquefied space, and at the same time a white film covers the surface of the solid or liquefied gelatine. With a napiform liquefaction the surface film remains indented round the point of inoculation. As the liquefaction proceeds downwards the original white cord like growth may persist more or less, or it 
may break up into floccules which slowly gravitate, leaving the upper liquefied gelatine clear, the lower layers turbid. The appearances are never like 36 , i. or 36 , ii., but approach 38 , iv. (Lehmann and Neumann, Diagnostik, i.). These appearances are similar with 15 and $20 \%$ nutrient gelatine.

Glucose-gelatine shake - There is no gas developed.

Agar plate.-The colonies are white and may assume various shapes. The surface colonies may be rounded with an irregularly lobed margin and a white central point where the surface growth has originated from a subsurface colony, or when the growth has always been upon the surface the centre may be dark. In cases where the agar surface is moist, the colony may send out amœboid processes which gradually extend over the whole surface after the manner of Bact. vulyare. The colonies just below the surface may send out processes into the agar medium. The organisms may also grow as a film between the solidified agar and the glass of the Petri dish. When examined with a 60 -fold magnification the colonies are seen to be highly granular. The margins of the surface colonies are sinuous or waved, and are seen to consist of filaments. The subsurface colonies are irregular in outline and beset with fibrous projections. The processes sent out by the subsurface colonies into the agar medium are seen to be composed of interlacing filaments.

Agar slope.-A matt, white expansion is quickly formed. It is slightly raised, and has an irregular margin which, when viewed with a lens, appears bristly. The expansion becomes folded or wrinkled, and approaches that figured in 38, viii. (Lehmann and Neumann). When touched with the needle the growth, when the medium is moist, breaks into crystal-like masses, or adheres, when the medium is dry, as a tough skin. When spore formation has set in, the growth becomes glistening, the folds or ridges become flatter, and when touched at this stage with the needle the consistency is found to be that of butter.

Agar stab.-The needle track develops an uncharacteristic filiform growth. The white surface growth spreads and becomes wrinkled or corrugated as 38, viii. (Lehmann and Neumann). 
Bouillon. - At first there appears a slight turbidity, which, while increasing, slowly gravitates, leaving the upper portions clear, the middle layers turbid, and a voluminous flocculent white precipitate at the bottom of the medium. A film grows upon the surface, and after a time becomes indented in places. There is no indol produced.

Potato.-A dry white layer is formed, which slowly spreads over the surface. It remains flat, and is never raised, puckered, nor wrinkled when grown at $22^{\circ}$. At $30^{\circ}$ a slight wrinkling may appear. The centre becomes of a slightly brown tinge, and in the old cultures (12 days) this brown colour has spread over the expansion.

Blood serum.-A spreading grey-white layer is formed on the solidified serum. The margin of the growth is irregular and bristly when viewed with a lens. The serum is not liquefied.

Milk.-The casein is first coagulated and then dissolved.

Germination. - For observing this process an old agar culture

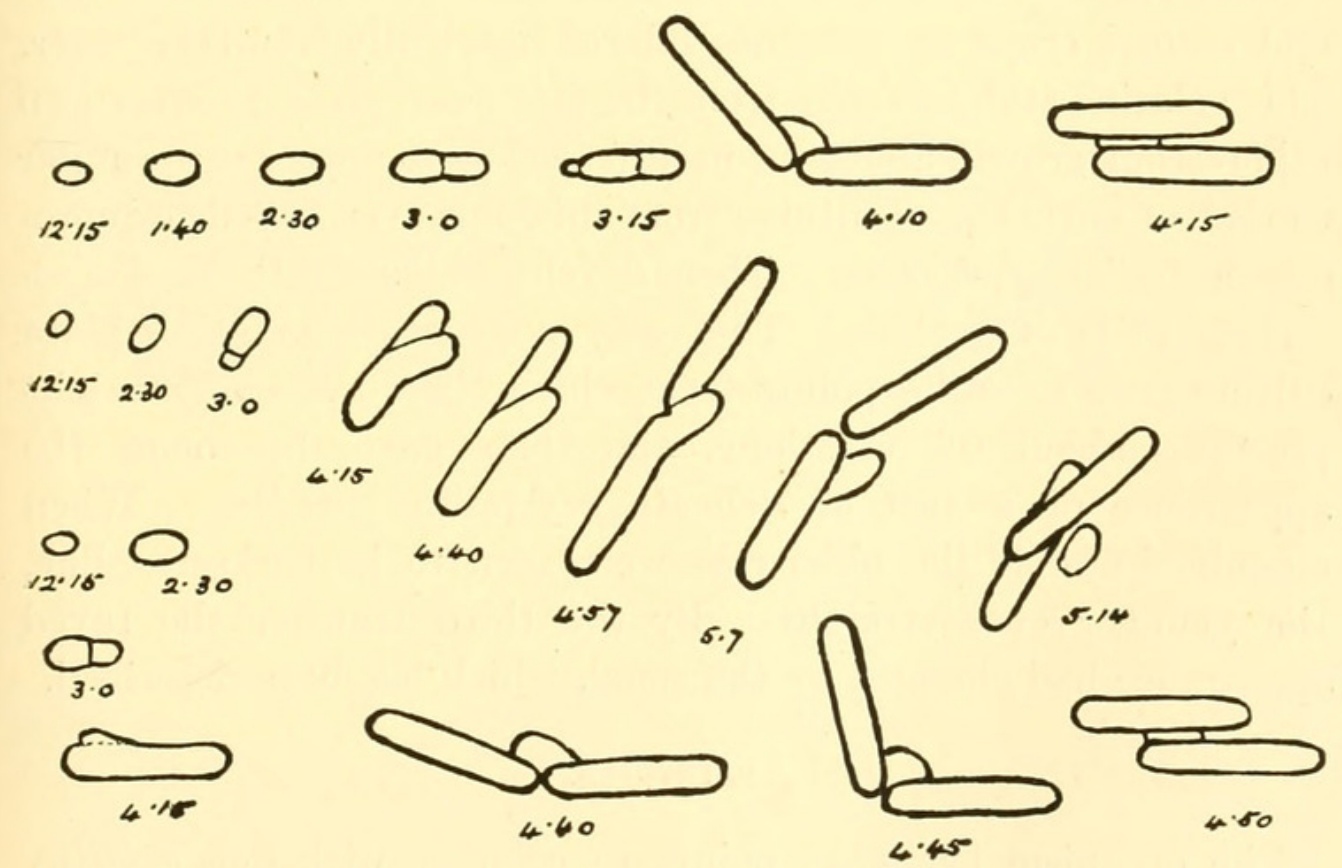

Germination of three spores in nutrient gelatine; the numbers indicate the times of observation.

was sown in gelatine and a loopful smeared over a sterile coverglass, which was then inverted over a hollow glass slide. The 
highly refractile spore slowly enlarged, and at the same time became less and less refractile. The rod emerged from one of the poles and grew outwards until the protruded end was as long as the spore case. By this time the distinction between rod and spore had practically disappeared. A protrusion then appeared at the other end of the spore case, and growth proceeded in two directions. The spore case was then thrown off, and this was followed by a division of the lengthened rod into two parts. The daughter bacillus, formed from the end last to emerge, slowly bent round until it was at an angle of $45^{\circ}$ with the other rod, when it suddenly slipped along the side of the latter. Germination occurred in $3 \frac{1}{2}$ hours at laboratory temperature $\left(20^{\circ}\right)$; an hour later there were two cells and in another, four bacilli. The dimensions of the mature rod after division and whilst thus imbedded in gelatine were $1.5: 7.5 \mu$.

The early gelatine cultures. - The characteristics that have been described appear to be permanent, for the characters of the twelfth transfer or crop were identical with the third. The first and second crops in gelatine differed markedly from the later. The gelatine stab infected from the first agar plate produced an arborescent growth along the needle track - an appearance similar to that of anthrax. Cultures from this, however, failed to reproduce a similar appearance. There developed instead the uncharacteristic filiform growth. The early colonies in gelatine plate culture grew as white points from which there radiated straight processes about $0.5 \mathrm{~cm}$. long, and these gave the colony the appearance of a tuft of delicate crystalline needles. When magnified 60 -fold the older rays were seen to be corkscrew-like, the younger ones straight. By the third transfer the rayed appearance had changed to the zonal, which has been described.

\section{Affinities.}

The organism has many points in common with Bac. subtilis, the common hay bacillus, but differs from it in the folded appearance in agar culture and the rosette-like folded processes of the young surface colonies in gelatine plate culture. Other differences 

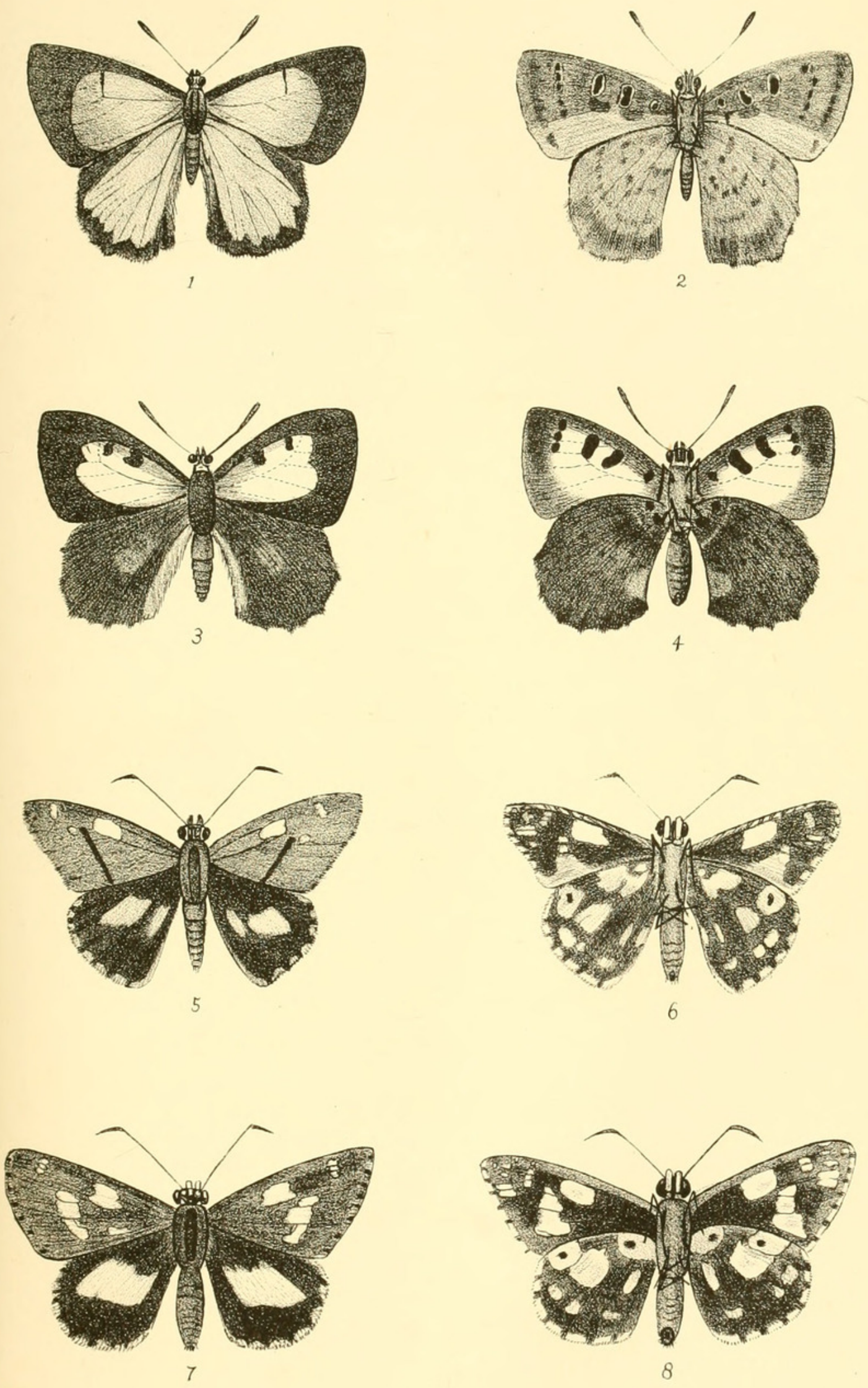

G.A.W. $\sigma_{B L}$. 



\section{$2 \mathrm{BHL}$ Biodiversity Heritage Library}

Greig-Smith, Robert. 1900. "A new bacillus pathogenic to fish." Proceedings of the Linnean Society of New South Wales 25, 122-130.

https://doi.org/10.5962/bhl.part.12154.

View This Item Online: https://www.biodiversitylibrary.org/item/30484

DOI: https://doi.org/10.5962/bhl.part.12154

Permalink: https://www.biodiversitylibrary.org/partpdf/12154

\section{Holding Institution}

MBLWHOI Library

Sponsored by

MBLWHOI Library

\section{Copyright \& Reuse}

Copyright Status: NOT_IN_COPYRIGHT

This document was created from content at the Biodiversity Heritage Library, the world's largest open access digital library for biodiversity literature and archives. Visit BHL at https://www.biodiversitylibrary.org. 